\title{
Microstrip Leaky-Wave Antenna With Control of Leakage Rate and Only One Main Beam in the Azimuthal Plane
}

\author{
José Luis Gómez-Tornero, Member, IEEE, David Cañete-Rebenaque, Student Member, IEEE, and \\ Alejandro Álvarez-Melcón, Member, IEEE
}

\begin{abstract}
An original leaky-wave antenna (LWA), conceived from a microstrip line which is laterally shielded by parallel plates, is presented in this paper. This structure shows, for the first time, that the leakage rate of a microstrip leaky-mode can be easily controlled while negligibly affecting the pointing direction. The antenna is based on the radiation from the second higher order mode of the microstrip line, which is perturbed by the addition of the two conductor walls to control the level of the leakage rate. The parallel-plates also serve as a mechanism to obtain a single main beam in the azimuthal plane, therefore improving the radiation pattern compared to common dual-beam second-higher order mode microstrip LWAs. The proposed antenna is analyzed by obtaining the corresponding leaky-mode complex propagation constant, which is calculated by a specific method of moments approach. The modal results obtained from the leaky-mode dispersion curves are validated with analysis performed on a three-dimensional structure using commercial finite element method solver. Also, a prototype is fabricated to experimentally confirm the advantages of this novel leaky-wave line source.
\end{abstract}

Index Terms-Laterally-shielded microstrip technology, leakage-rate control, leaky-wave antennas (LWAs).

\section{INTRODUCTION}

$\mathbf{M}$ ICROSTRIP lines have been used to conceive planar leaky-wave antennas (LWAs), after it was experimentally demonstrated in 1979 the ability of higher order modes to radiate close to the cutoff regime [1]. These results created much interest to study the radiation properties of higher order modes in printed lines, in order to synthesize planar leaky-wave line sources [2], [3]. The complex propagation constant of the leaky-modes must be obtained to identify the radiation region and to design the microstrip line dimensions to operate in a certain frequency band [3]-[6]. Most of microstrip leaky-wave antennas (MLWAs) are based on the radiation of the first higher order mode ([1]-[12]). Different feeding networks have been proposed for this antenna [8]-[11]. Moreover, this MLWA has

Manuscript received October 31, 2006; revised October 10, 2007. This work was supported in part by the Spanish National project TEC2004-04313-C02-02/ TCM, in part by the Regional Seneca project 02972/PI/05, and in part by Regional Scholarship PMPDI-UPCT-2007.

The authors are with the Departamento de Tecnologías de la Información y las Comunicaciones, Universidad Politécnica de Cartagena, Cartagena 30202, Spain (e-mail: josel.gomez@upct.es).

Color versions of one or more of the figures in this paper are available online at http://ieeexplore.ieee.org.

Digital Object Identifier 10.1109/TAP.2007.915422 also been applied to conceive planar active integrated antennas [12]. The second higher order mode of the microstrip line has also been proposed to design another type of MLWA [13]. Different works have been done to efficiently excite this mode [14]-[16].

In any case, all the previously studied MLWAs have a very important design disadvantage: the leakage rate of the corresponding leaky-mode cannot be freely controlled [10]-[15]. As it is well known, one needs to independently control the phase and the leakage rate of a leaky-mode in order to freely design the radiation properties of a LWA [17]. Particularly, the phase constant allows to choose the pointing direction of the main radiated beam, while the control on the leakage rate allows to select the radiation efficiency and beamwidth, and to design a tapered radiation pattern with a given sidelobes distribution [17]. In this paper, we propose for the first time an original mechanism to control the leakage constant of the second higher order mode of the MLWA by introducing parallel plates in both sides of the microstrip line. This mechanism also allows to obtain a single main beam in the azimuthal plane, therefore avoiding the dual-beam radiation pattern associated to second-higher order MLWAs [13]-[16]. In Section II, the proposed structure is introduced, and the basic theoretical concepts to understand the radiation mechanism are explained in detail. Using the leaky-mode dispersion curves, the advantages of this novel LWA are shown in a practical design operating at $6 \mathrm{GHz}$. Finite element method (FEM) analysis of the structure are used to validate the theory. Finally, a prototype is fabricated and measured in Section III to experimentally check the electrical advantages of the new antenna.

\section{The LATERALLY-ShIELDED MicrostRIP LiNE}

Fig. 1 shows the cross section of the structure studied in this paper. It is a microstrip line of width $W$, printed on a substrate of height $H$ and relative permittivity $\varepsilon_{r}$, and laterally shielded by parallel-plates of height $H+L$, separated at a distance $a$ [see dimensions in Fig. 1(a)]. The transverse electric field lines of different types of modes of this structure are shown in Fig. 1. Fig. 1(a) represents the fundamental mode of the microstrip line, which is always bound for the proposed structure. In Fig. 1(b), the first higher order mode is illustrated. This mode has a leaky-wave regime, which has been extensively studied to conceive MLWAs [1]-[11]. As it is illustrated in Fig. 1(b), the field lines radiate out from the microstrip line, with little influence of the lateral metallic walls. Finally, Fig. 1(c) and 


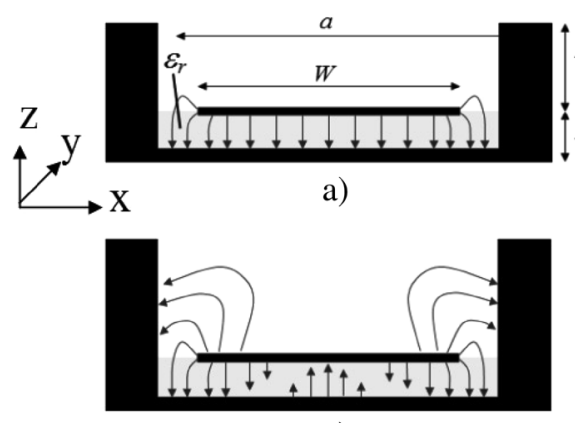

c)

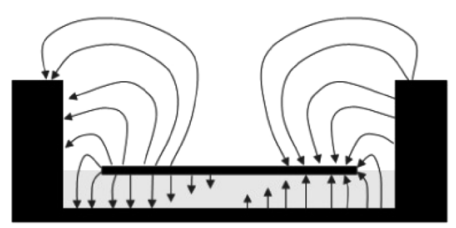

b)

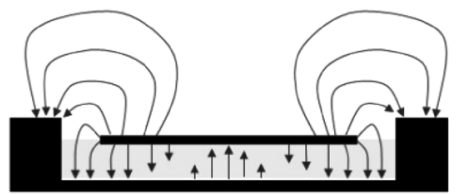

d)

Fig. 1. Electric field lines of different modes in the laterally-shielded microstrip line.
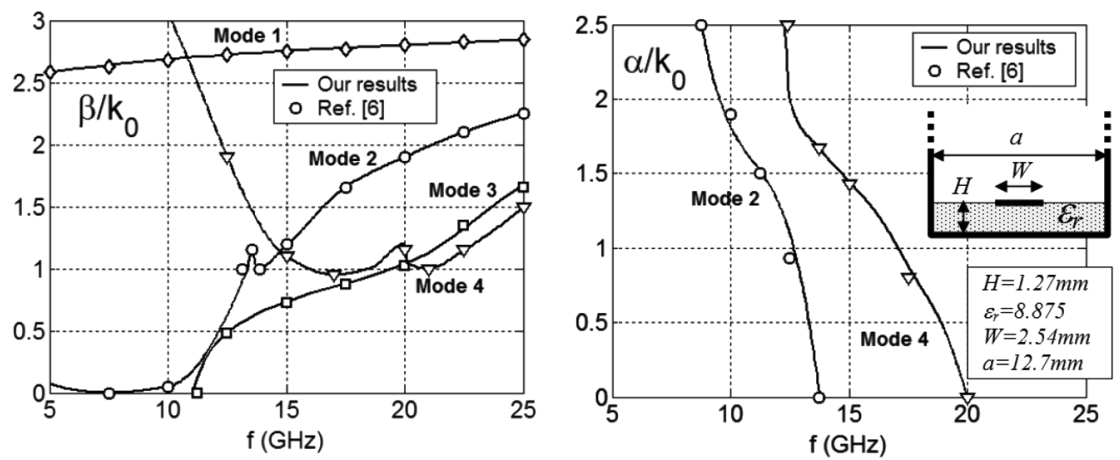

Fig. 2. Normalized phase and attenuation constant for the first four modes in a laterally-shielded microstrip line with lateral walls of infinite height.

Fig. 1(d) represent the second higher order mode of the laterally-shielded microstrip line. In opposition to what happens to the first higher order mode, the radiation of the second-higher order mode strongly depends on the conductor lateral walls. As it will be demonstrated, the mode will not radiate if the sidewalls are high enough [see Fig. 1(c)], since the electromagnetic energy cannot reach the top aperture. On the contrary, if the lateral walls are shortened [see Fig. 1(d)], the fields can reach the top of the structure, and the mode will have a leaky-wave regime. If the lateral plates are completely removed, we have the case of the common microstrip line operated in its second higher order mode, used in previous works to conceive dual-beam MLWAs [13]-[16].

To understand this difference between the behavior of the first and the second higher order modes of the shielded microstrip line, we can use the expansion of the total fields as a sum of parallel-plate harmonics (PPHs) [18]. The fields of any permitted mode in the laterally-shielded microstrip line can be decomposed as a sum of infinite PPHs. This is the basis of the Greens functions developed in [18] and [19] to analyze this type of structures using the method of moments (MoM). The propagation constant in the transverse z-direction of the parallel-plate region [see Fig. 1(a)] for any PPH of order " $m$ " can be expressed as [18]

$$
k_{z}^{\mathrm{PPH} m}=\sqrt{k_{0}^{2}-\left(m \frac{\pi}{a}\right)^{2}-\beta^{2}}
$$

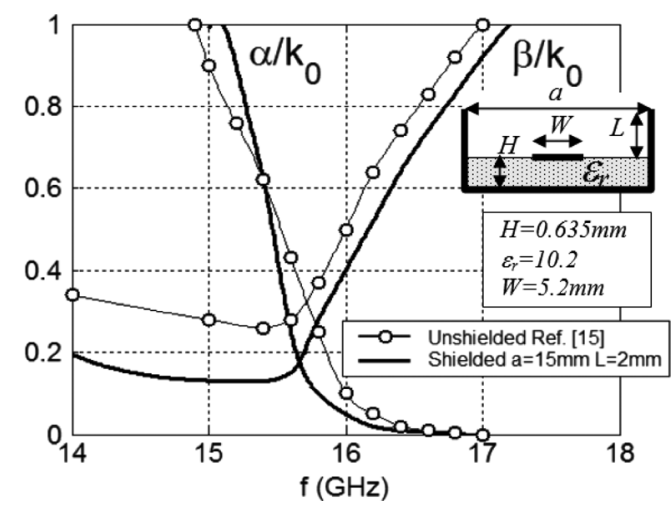

Fig. 3. Comparisons between unshielded and shielded microstrip line.

where $\beta$ is the phase constant of the studied leaky-mode in the longitudinal direction of the microstrip structure [y-axis, see Fig. 1(a)]. The first higher order mode of the laterally-shielded microstrip line strongly excites the PPH of order $m=0$, due to its odd symmetry [see Fig. 1(b)]. This space-harmonic of order $m=0$ can propagate along the parallel-plates, from the microstrip line to the top aperture, since its transverse propagation constant $\left(k_{z}\right.$ with $\left.m=0\right)$ is real in the fast-wave region of the microstrip mode (when $\beta / k_{0}<1$ ). Therefore, the first higher order mode of the microstrip line can radiate, no matter the height of the parallel-plates, as it was illustrated in Fig. 1(b). On the contrary, the second higher order mode of the microstrip 


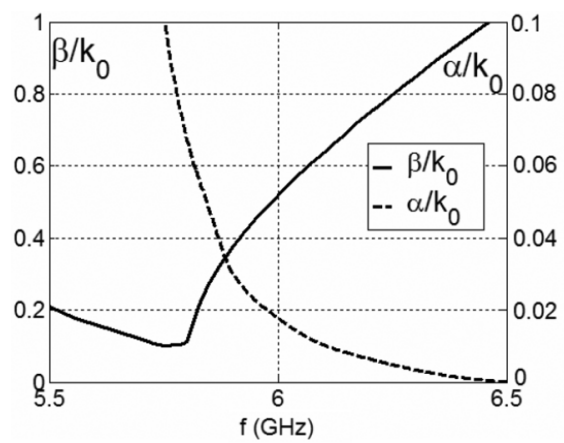

a)

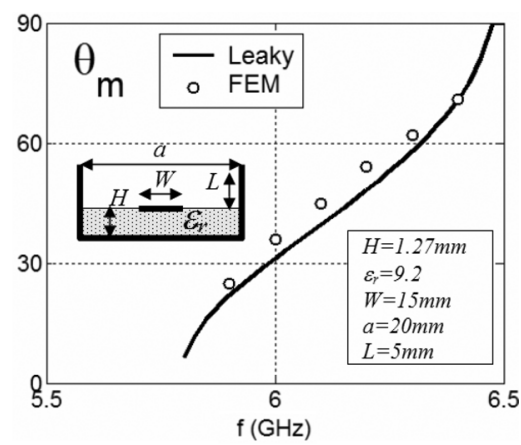

b)

Fig. 4. Dispersion curves for the microstrip second higher order mode in the proposed structure: (a) normalized phase and attenuation constants and (b) elevation pointing direction.

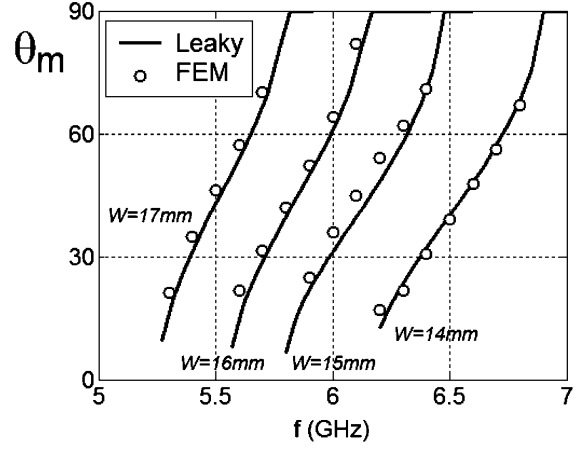

a)

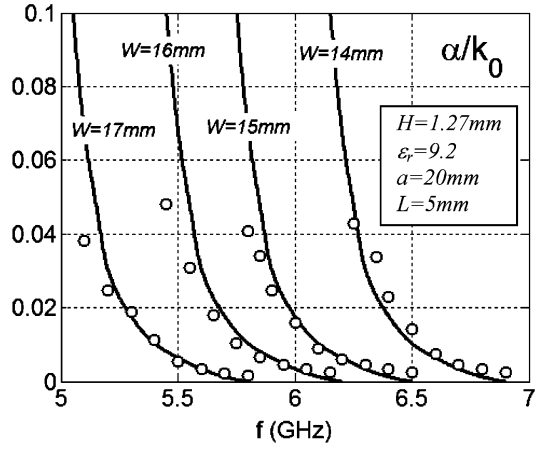

b)

Fig. 5. Influence of the strip width $W$ on the leaky-mode frequency-dispersion curves

does not excite the PPH of order $m=0$ due to its even symmetry [see Fig. 1(c)]. This second higher order mode can therefore be decomposed as a sum of PPH with $m>0$. The parallel-plates act as a filtering mechanism that does not allow these PPH to freely propagate in the z-transverse direction, preventing the radiation if the distance between the microstrip and the top aperture ( $L$, in Fig. 1$)$ is high enough. To assure this evanescent nature for all higher order PPH $(m>0)$, their transverse propagation constant in the parallel-plate region $k_{z}$ (1) must be imaginary (so that these PPHs are below cutoff in the parallel-plate region). It can be easily demonstrated that this condition is assured if the distance between the parallel-plates " $a$ " is shorter than half free-space wavelength, $\lambda_{0}$ [20]

$$
a<\frac{\lambda_{0}}{2} \rightarrow a(m m)<\frac{150}{f(\mathrm{GHz})}
$$

As an example, Fig. 2 shows the normalized phase and attenuation constants $\left(\beta / k_{0}\right.$ and $\alpha / k_{0}$, respectively) as a function of frequency, for the first four modes in a laterally shielded microstrip line with infinite sidewalls studied in [6]. Our results are obtained with the MoM approach developed in [18], [19], and are compared with the results obtained in [6] with a mode matching technique. Excellent agreement is observed between the two techniques. Since the parallel-plates of the analyzed structure have infinite height $(L=\infty)$, the second higher order mode (Mode 3 in Fig. 2) does not radiate, therefore not having leakage constant $\left(\alpha / k_{0}=0\right)$ in its fast-wave region
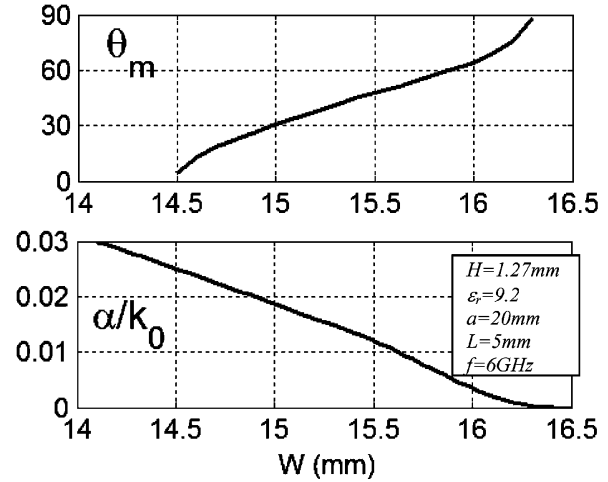

Fig. 6. Control of the pointing direction for a fixed frequency $(6 \mathrm{GHz})$ by modifying the strip width $W$, and its effect on the leaky-mode attenuation rate.

$\left(\beta / k_{0}<1\right)$. On the contrary, the first-higher order mode (Mode 2 in Fig. 2) does radiate even with the presence of infinite parallel-plates due to its odd symmetry, as it happens for the third higher order mode (Mode 4 in Fig. 2).

Another illustrative numerical example is shown in Fig. 3, where the frequency dispersion curves for the second higher order mode of a microstrip line studied in [15] are plotted. The results of [15] were obtained for an unshielded microstrip line using a Spectral Domain technique, and are plotted with circles. Our results are plotted with continuous line, and are for the same structure, but with parallel-plates of height $L=2 \mathrm{~mm}$, separated a distance $a=15 \mathrm{~mm}$. In this case, the second higher 


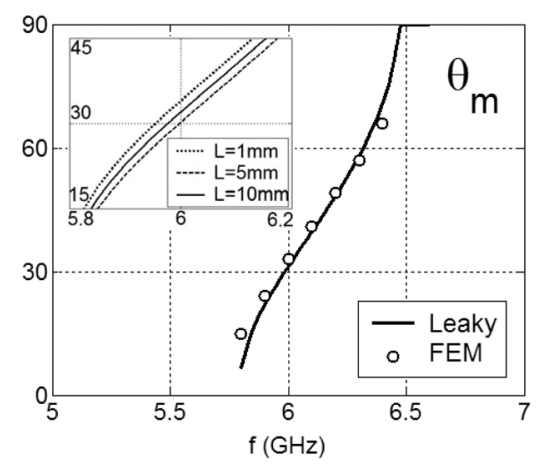

a)

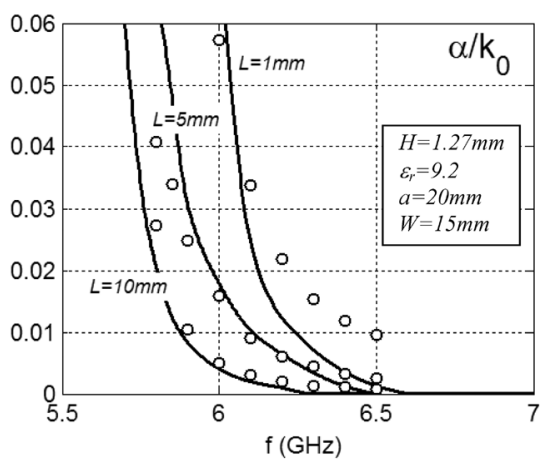

b)

Fig. 7. Influence of the parallel-plates height $L$ on the leaky-mode frequency-dispersion curves.

order mode of the microstrip line can radiate in its fast-wave region $\left(\beta / k_{0}<1\right.$, below $\left.17 \mathrm{GHz}\right)$, since the parallel-plates are short enough. We will call this radiating structure a laterallyshielded microstrip leaky-wave antenna (LSMLWA). Moreover, in Fig. 3 we can see the influence of the sidewalls, if we compare our results with those obtained for the unshielded microstrip line in [15]. As it can be seen, the two structures behave similarly, but a lower leakage rate is observed in the laterally-shielded antenna due the effect of the walls. We can also observe a shift to higher frequencies in the phase constant curve.

From the results shown in Fig. 2 and Fig. 3, we can conclude that the leakage rate of the second higher order mode of a microstrip line can be eliminated if parallel-plates of enough height $L$ are located at a distance $a<\lambda_{0} / 2$. A last condition must be satisfied for a proper operation. We must take into account that the second higher order mode of the microstrip line must be above cutoff to propagate along the microstrip axis. For this purpose, this mode must resonate in the cross-section of the microstrip of width $W$, having the following wavenumber in the $\mathrm{x}$-direction (see axes in Fig. 1)

$$
k_{x}^{\mathrm{STRIP}} \approx \frac{2 \pi}{W} .
$$

From the wavenumber condition in the dielectric slab we can obtain the approximate minimum strip width to allow this second higher order mode to propagate

$$
\begin{aligned}
k_{0}^{2} \varepsilon_{r} & =k_{x}^{2}+\beta^{2}=\left(\frac{2 \pi}{W}\right)^{2}+\beta^{2} \\
\beta^{2} & >0 \rightarrow W(m m)>\frac{300}{f(\mathrm{GHz})} \frac{1}{\sqrt{\varepsilon_{r}}} .
\end{aligned}
$$

Since the parallel-plates separation $a$ must be greater than the strip width $W$, and combining the conditions of (2) and (5), we finally obtain

$$
\begin{aligned}
a_{\mathrm{MAX}}(m m) & =\frac{150}{f(\mathrm{GHz})}>W_{\mathrm{MIN}}(m m) \\
& >\frac{300}{f(\mathrm{GHz})} \frac{1}{\sqrt{\varepsilon_{r}}} \rightarrow \varepsilon_{r}>4 .
\end{aligned}
$$

This condition establishes a minimum relative permittivity for the dielectric used in this type of LSMLWA. With this condition, it will be demonstrated that the parallel-plates can control the leakage rate of the second higher order mode microstrip line, while negligibly affecting its phase constant. Moreover, it will be shown the ability to obtain a single main radiated beam in the azimuthal plane.

\section{A. Control of Complex Propagation Constant}

The proposed mechanism to control the leakage rate will be checked by designing a LSMLWA operating in its second higher order mode. A commercial dielectric substrate (Rogers TMM 10) with $H=1.27 \mathrm{~mm}, \varepsilon_{r}=9.2, \tan \delta=0.0022$ will be used to support the microstrip line. This substrate satisfies the condition imposed in (6). The width of the strip is chosen to be $W=15 \mathrm{~mm}$ in order to operate with a fast-wave second-higher order microstrip mode around $6 \mathrm{GHz}$. According to (2), the maximum distance between the parallel-plates at this frequency must be $a_{\mathrm{MAX}}=25 \mathrm{~mm}$, in order to generate evanescent fields in the dielectric-parallel-plate interface. At is has been explained, this condition is necessary to control the leakage rate of the leaky-wave. For this reason, sidewalls separated at a distance $a=20 \mathrm{~mm}$ will be used in the proposed LSMLWA.

Fig. 4(a) shows the dispersion curves obtained with the leakymode MoM approach. As it can be seen, the leaky-wave band is between 5.7 and $6.5 \mathrm{GHz}$. In Fig. 4(b), the elevation pointing angle measured from the broadside direction $\left(\theta_{m}\right)$ is extracted from the leaky-mode phase constant $\left(\beta / k_{0}=\sin \theta_{m}\right)$, and it is compared with the results obtained from finite element method (FEM) simulations using a commercial solver. Good agreement is observed between the leaky-mode technique and the analysis of the three dimensional structure (FEM) for the frequency scanning response. We can see that the leaky-mode radiates around $\theta_{m}=34^{\circ}$ for the design frequency of $6 \mathrm{GHz}$.

Apart from the frequency-beam-scanning capability of this LWA [shown in Fig. 4(b)], it is interesting to control the pointing direction for the desired frequency. As it is well-known [3], [4], for the case of MLWAs this can be done by adjusting the strip width $W$, as it is shown in Fig. 5. In this figure, the elevation pointing direction and the leakage rate are represented as a function of frequency, for four different strip widths around the reference value of $W=15 \mathrm{~mm}$. As it can be seen in Fig. 5, the cutoff frequency of the microstrip second-higher order-mode 


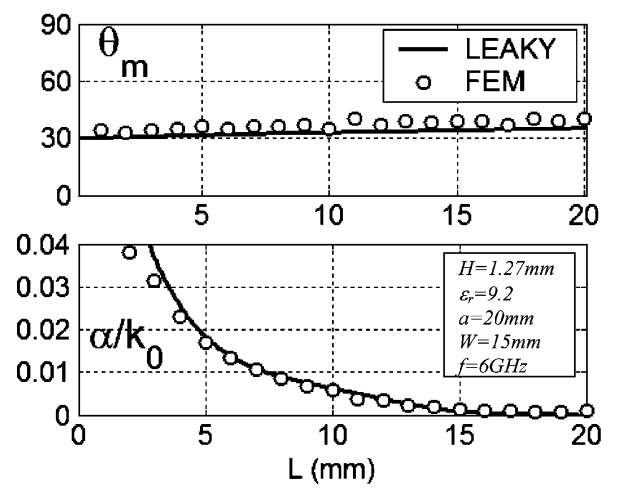

Fig. 8. Control of the leakage-rate for a fixed frequency $(6 \mathrm{GHz})$ by modifying the parallel-plates height $L$, and its effect on the leaky-mode pointing direction.

decreases as $W$ is increased. In this way, the pointing direction $\theta_{m}$ of the MLWA can be increased for a fixed frequency when the strip is made wider, as it is illustrated in Fig. 6 for a design frequency of $6 \mathrm{GHz}$. However, the modification of $W$ has also a strong influence on the leakage-rate, as it can be seen by the attenuation-rate frequency dispersion curves shown in Fig. 5(b). This is again due to the fact that a modification of $W$ involves a change in the cutoff frequency of the leaky-mode, and therefore a strong change in the whole complex propagation constant. As it can be seen in Fig. 5, both $\theta_{m}$ and $\alpha / k_{0}$ dispersion curves are shifted together to lower frequencies as $W$ is increased.

As a result, we can conclude that the strip width $W$ allows to tune the pointing direction of the LWA, with the drawback that the associated leakage-rate will also be strongly modified, as it is shown in Fig. 6. This is a general situation for all MLWAs, in which the designer cannot independently select the pointing direction and the leakage-rate for a given substrate and frequency of operation.

As it has been previously commented, once the pointing direction has been chosen, it is necessary to find a way to modify the leakage rate in order to synthesize a desired radiation pattern (with a given radiation efficiency and main beam width), while negligibly affecting the selected pointing direction [17]. For this purpose, the parallel-plates height $L$ can be modified, as it is illustrated in Fig. 7. In this figure, the frequency dispersion curves for both the pointing direction and the leakage rate of the leaky-mode are represented, for three different values of $L$. As it was explained in the introduction section, the parallel-plates act as a filtering circuit which make the fields of the second-higher order mode of the microstrip line to be evanescent in the air-dielectric interface, provided that (2) and (6) are satisfied. In this way, we are able to reduce the radiation as the height $L$ is increased, as it can be seen in the leakage-rate curves shown in Fig. 7(b). Moreover, it is important to notice that the leaky-mode pointing angle dispersion curves [shown in the inset of Fig. 7(a)] barely change when altering the parallel-plates height $L$. This is due to the fact that the cutoff frequency of the microstrip leaky-mode does not strongly depend on $L$, in opposition to what happened when the strip width $W$ was modified (see Fig. 5). As it is shown in Fig. 7, good agreement is again observed between leaky-mode analysis and results obtained with FEM analysis of the three-dimensional leaky-wave structure.
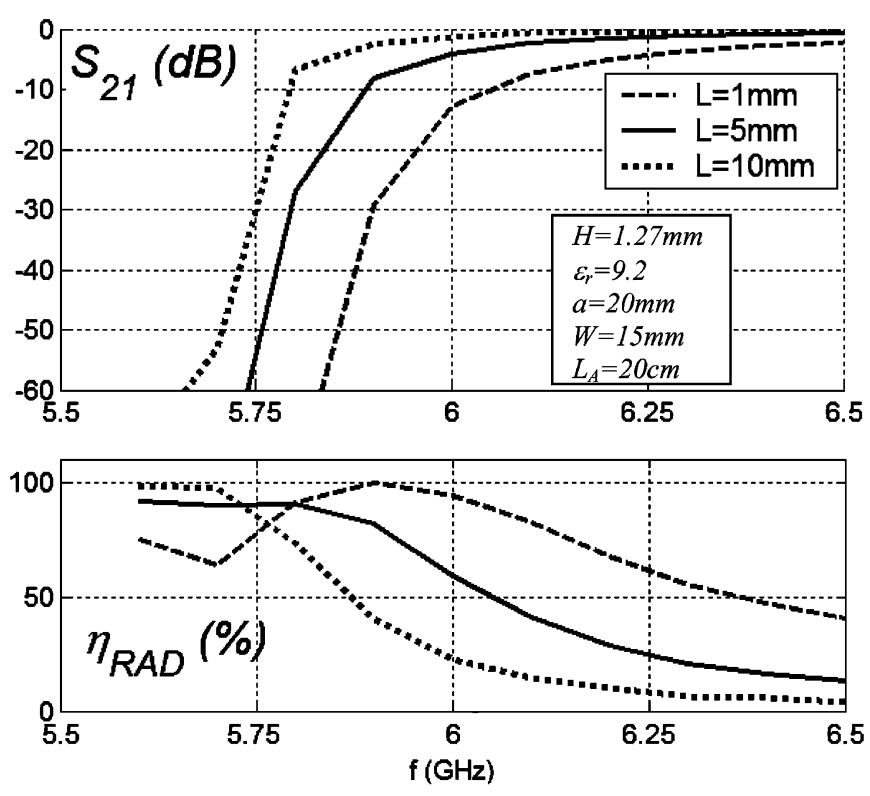

Fig. 9. Control of the radiation efficiency by modifying the parallel-plates height $L$.

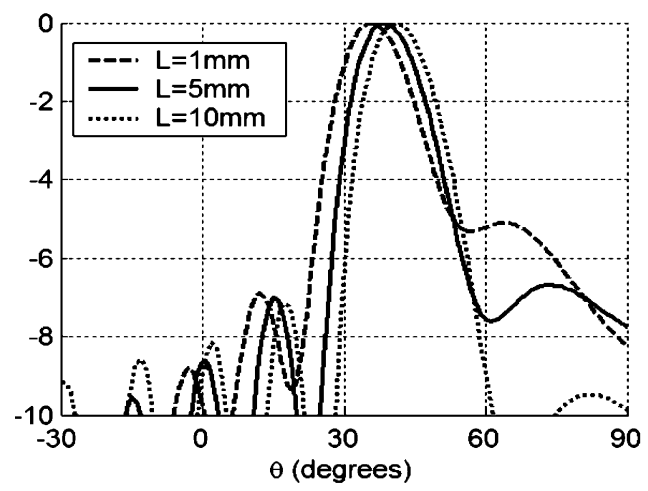

Fig. 10. Normalized radiation patterns in the elevation plane at $6 \mathrm{GHz}$, for different heights of the parallel-plates.

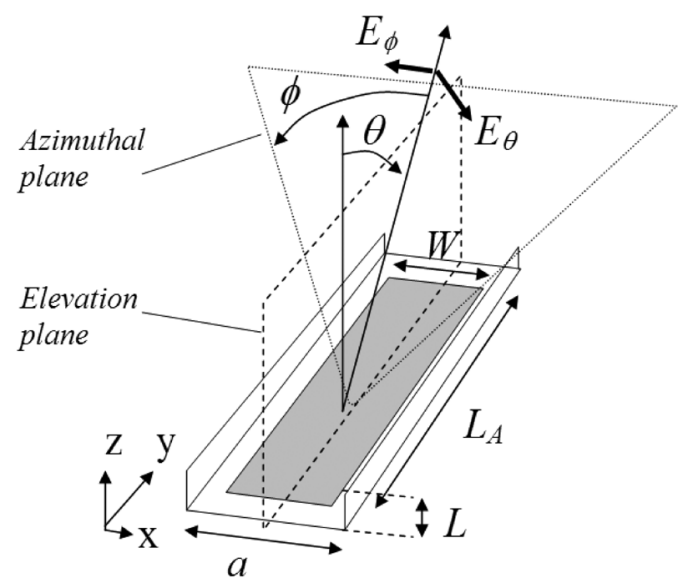

Fig. 11. Main dimensions and references axis used in the novel LSMLWA.

Therefore, we can use the parallel-plates height $L$ to control the leakage rate, once the pointing direction has been chosen for a fixed frequency by properly designing the strip width $W$ for a given substrate. Fig. 8 shows the leakage-rate design curves 

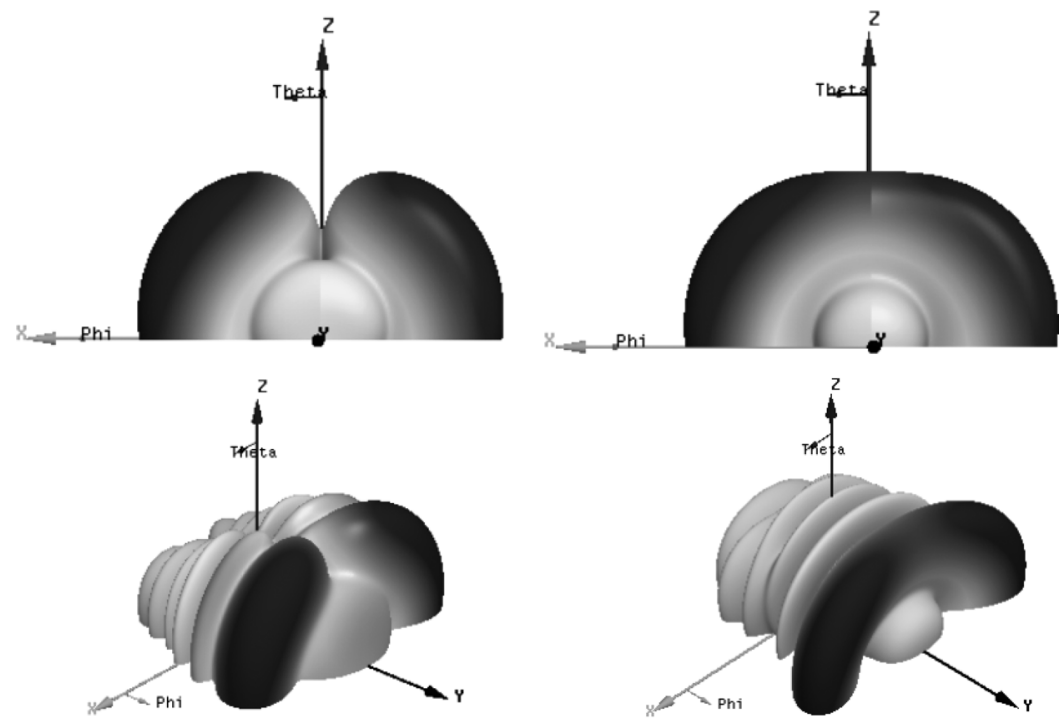

a)

b)

Fig. 12. Effect of the parallel-plates in the radiation diagram of a MLWA. (a) No parallel-plates and (b) parallel-plates with $L=10 \mathrm{~mm}$.

at $6 \mathrm{GHz}$ obtained for a strip of width $W=15 \mathrm{~mm}$, which gives raise to a pointing angle around $\theta_{m}=30^{\circ}$ for the selected substrate (as it was shown in Fig. 6). As it corresponds to reactive fields, exponential decrease of $\alpha$ is obtained when $L$ is increased. The variation of the pointing angle with $L$ has also been studied, and it is plotted in Fig. 8. We observe that $\theta_{m}$ is slightly affected when $L$ is modified. Good agreement between leaky-mode analysis and FEM results is observed for these dispersion curves, confirming the theory to control the leakage rate with small influence on the pointing direction.

An important practical application of this control of the leakage rate is that one can freely choose the radiation efficiency of the LWA for a given antenna length (which determines the main beam width) [17]. Alternatively, one can also modify the beamwidth by selecting the antenna length and the appropriate leakage rate, so that the radiation efficiency is maximized (normally to $90 \%,[17])$. In any case, it is necessary to keep the desired main beam pointing direction while choosing the appropriate leakage-rate. The following results, obtained from FEM analysis of the proposed LSMLWA, illustrate the advantage of this novel LWA (compared to previous MLWA in which the leakage rate could not be chosen independently of the pointing direction). The antenna length is chosen to be $L_{A}=20 \mathrm{~cm}$ (which is $4 \lambda_{0}$ at $6 \mathrm{GHz}$ ), and the dimensions of the cross section of the laterally-shielded microstrip line are the same than in previous graphs $\left(H=1.27 \mathrm{~mm}, \varepsilon_{r}=9.2\right.$, $a=20 \mathrm{~mm}, W=15 \mathrm{~mm}$ ). From the FEM analysis of the 3D LWA, the S parameters are obtained, and the radiation efficiency can be extracted assuming a lossless scenario

$$
\eta_{R A D}=1-\left|S_{21}\right|^{2}-\left|S_{11}\right|^{2} .
$$

Fig. 9 show the $\mathrm{S}$ parameters and the extracted radiation efficiency of the studied LWA structure, by modifying the height of the parallel-plates. Only the $S_{21}$ parameter is shown, since perfect matching is assumed by using a waveport to excite the second-higher order-mode of the microstrip-line.

As it can be seen in Fig. 9, $S_{21}$ decreases as the parallel-plates are shortened, due to the increase in the leakage-rate. In this way, for the design frequency of $6 \mathrm{GHz}$, the radiation efficiency can be modified from $22 \%$ (for $L=10 \mathrm{~mm}$ ) to $95 \%$ (for $L=1 \mathrm{~mm}$ ). Finally, Fig. 10 shows the FEM simulated normalized radiation pattern of the LSMLWA in the elevation plane $(\theta$ is the elevation angle measured with respect to the broadside direction, see Fig. 11), for different values of $L$. As it can be seen, the designed pointing angle $\left(\theta_{m}=34^{\circ}\right)$ is barely perturbed when the parallel-plate height is modified.

\section{B. Radiation Pattern in the Azimuthal Plane}

Another important advantage of the novel LSMLWA involves its radiation pattern in the azimuthal plane (for a definition of references planes, see Fig. 11). As it is well-known, MLWAs based on the second-higher order-mode of the microstrip line present a dual-beam in the azimuthal plane ([13]-[16]). The three-dimensional radiation pattern for a conventional (without parallel-plates) second-higher order-mode MLWA is shown in Fig. 12(a). This result was obtained with FEM for the same LWA studied in previous sections, but removing the parallel-plates. This dual-beam can be an undesired effect, since a relative minimum of radiation occurs in the y-axis of the antenna, as it is shown in Fig. 12(a). The novel LSMLWA presents a single radiated beam in the orthogonal plane, as it is illustrated in Fig. 12(b), where the same LWA but now with parallel-plates of height $L=10 \mathrm{~mm}$ has been analyzed with FEM code.

This strong difference between the laterally-shielded and the unshielded MLWA azimuthal radiation patterns can be explained by inspecting the near fields of the novel LSMLWA, which are shown in Fig. 13.

Fig. 13(a) illustrates the transverse electric field in the cross section of the proposed LSMLWA (Z-X plane, see Fig. 1), 


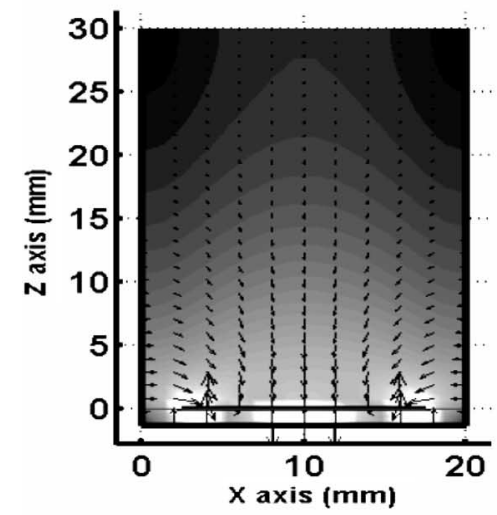

a)

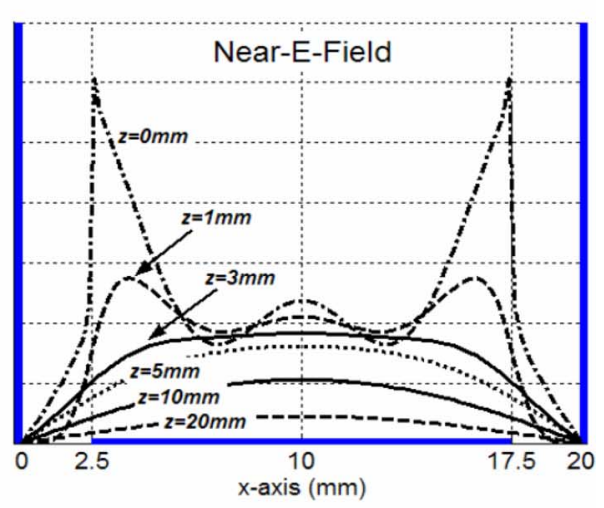

b)

Fig. 13. Near electric field plot for the novel LSMLWA: (a) field distribution in the cross-section and (b) field intensity along x-direction for different height $z$ above the microstrip line.

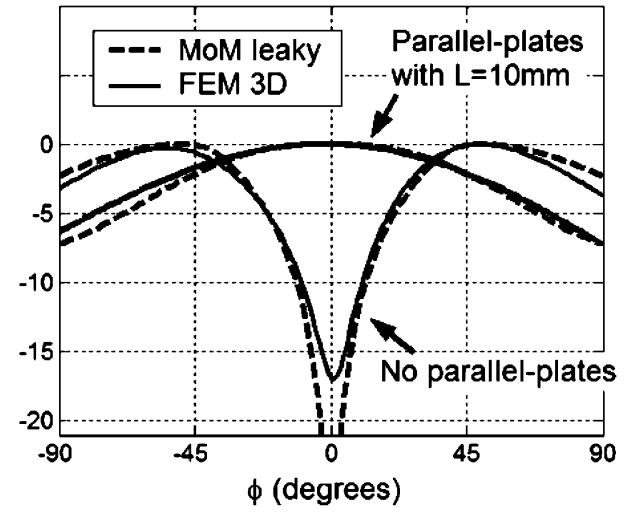

a)

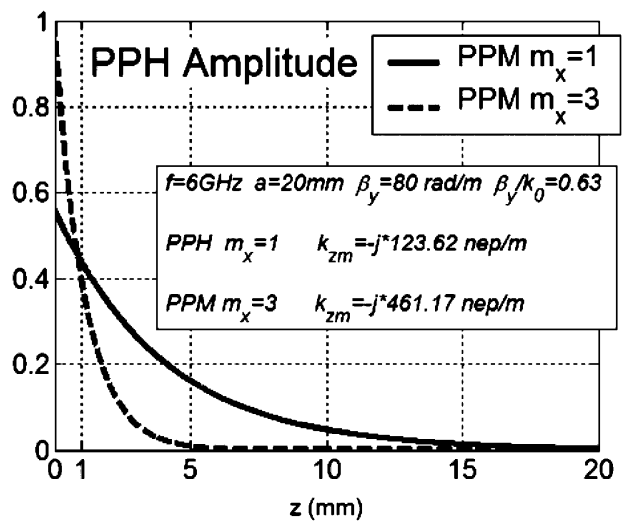

b)

Fig. 14. (a) Normalized azimuthal radiation pattern showing the effect of the parallel-plates and (b) evolution of parallel-plate higher order-modes along the z-direction.

obtained with MoM leaky-wave analysis. In Fig. 13(b), one-dimensional cuts of the field intensity along the $\mathrm{x}$-direction are represented for different heights $z$ above the microstrip line. Close to the microstrip line, the electric field pattern is described by the second-higher order harmonic of the microstrip line, which presents two maxima at the strip edges, as it is illustrated in Fig. 13(b) for $z=0$. If this second-higher order fields could directly radiate into free space, a dual-beam radiation pattern in the azimuthal plane would be created, as it happens in conventional second-higher order mode MLWAs. However, the effect of the parallel-plates is to filter this higher order variation of the fields as we move along the parallel-plates height (z-direction), from the microstrip interface $(z=0)$ to the parallel-plates aperture $(z=L)$. As it can be seen in Fig. 13, the near field distribution tends to present one single maximum (located in the middle of the two parallel-plates) for higher values of $z$. In this way, only this first-harmonic variation of the fields reach the top radiating aperture, located at a height $L$ from the microstrip line. These fields create a single radiated beam in the azimuthal plane, in opposition to the dual-beam created by the higher order harmonics close to the microstrip line. This is checked in Fig. 14(a), where the radiation pattern has been obtained by taking the Fourier transform of the near fields (computed with the MoM leaky-mode analysis technique) for two cases: presence of parallel-plates (with $L=10 \mathrm{~mm}$ ), and absence of parallel-plates. The results are compared with FEM analysis, confirming that the parallel-plates are responsible for the appearance of one single beam in the azimuthal radiation pattern.

The filtering response of the parallel-plates can easily be explained in terms of the parallel-plates harmonics (PPHs) which are excited in the parallel-plate region [18]. Using the MoM technique, we can obtain the PPHs excited by the second-higher order leaky-mode of the microstrip line. The third-order PPH [ $m=3$ in (1)] is the more excited PPH, followed by the firstorder PPH $[m=1$ in (1)], as it is illustrated in Fig. 14(b). In this figure, the evolution of the amplitude of these PPH along the parallel-plates transverse z-direction is represented $(z=0$ corresponds to the microstrip-air interface). As described in the introduction of this work, the transverse wavenumber $k_{z m}$ for each PPH can be calculated using (1). For $f=6 \mathrm{GHz}$ and the computed longitudinal wavenumber $(\beta=80 \mathrm{rad} / \mathrm{m})$, we obtain the values of $k_{z m}$ showed in the inset of Fig. 14(b) for these two main PPHs. As expected, the third-order PPH is much more evanescent than the first-order PPH, therefore presenting a higher attenuation constant in the z-direction. Due to the higher reactive nature of the third-order PPH, the first-order PPH becomes predominant as we move far from the microstrip line. We can see in Fig. 14(b) how most of the energy is carried only by the first-order PPH for $z>2 \mathrm{~mm}$. In this way, only the first-order PPH reach the top radiating aperture $(z=L)$, avoiding the dual-bam azimuthal radiation pattern associated 


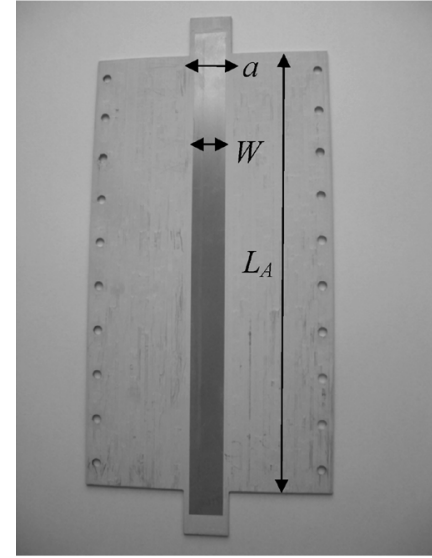

a)

d)

INPUT
(1)

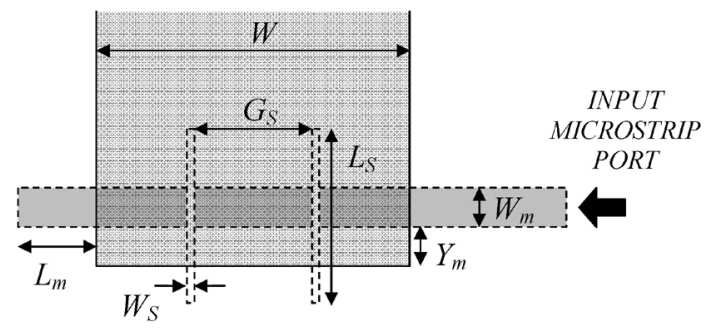

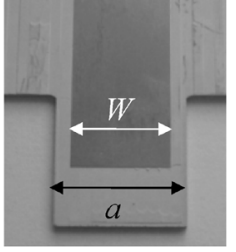

b)

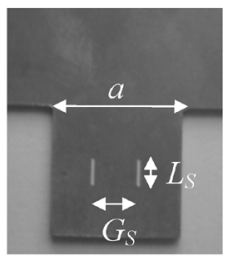

c) trip LWA

Fig. 15. (a) Photograph of fabricated second-higher order microstrip LWA. (b)-(d) Detail of aperture-coupled excitation circuit.

to the third-order $\mathrm{PPH}$, and creating a single main beam as shown in Fig. 14(a). Moreover, the exponential decay of the fields shown in Fig. 14(b) suggests the control of the leakagerate by modifying the parallel-plates height $L$. As it can be seen in Fig. 14(b), no radiation should be obtained over $L=20 \mathrm{~mm}$, since the fields amplitude is negligible for this height. As we reduce the parallel-plates heights, increasing radiation should be obtained. This statement is in perfect accordance with the results shown in Fig. 8 for the dependence of the leakage-rate with $L$.

\section{FABRICATION AND MEASUREMENTS}

A prototype of the proposed LSMLWA has been fabricated using Rogers substrate TMM $10\left(H=1.27 \mathrm{~mm}, \varepsilon_{r}=9.2\right.$, $\tan \delta=0.0022$ ), and a microstrip line of width $W=15 \mathrm{~mm}$ and length $L_{A}=20 \mathrm{~cm}$, as it is shown in Fig. 15(a). The second higher order mode of the microstrip line is excited using the aperture-coupled technique described in [16], also illustrated in Fig. 15. The dimensions of the feeding circuit, according to the scheme of Fig. 15(d) are: $W=15 \mathrm{~mm}, a=20 \mathrm{~mm}, W m=$ $1.4 \mathrm{~mm}, L m=6.7 \mathrm{~mm}, W s=0.4 \mathrm{~mm}, L s=4.3 \mathrm{~mm}, G s=$ $7.0 \mathrm{~mm}$, and $Y_{m}=2 \mathrm{~mm}$. A robust and modular structure has been fabricated, so that parallel-plates of different height $L$ can be added to the microstrip antenna, in order to conceive the proposed LSMLWA. The distance between the parallel-plates is fixed to $a=20 \mathrm{~mm}$. A photograph of the LSMLWA prototype is shown in Fig. 16.

The measured S-parameters of the constructed prototype as a function of frequency are shown in Fig. 17 for different parallel-plates heights $L$. As it can be seen, good matching is observed for the designed frequency and for any value of $L$. From

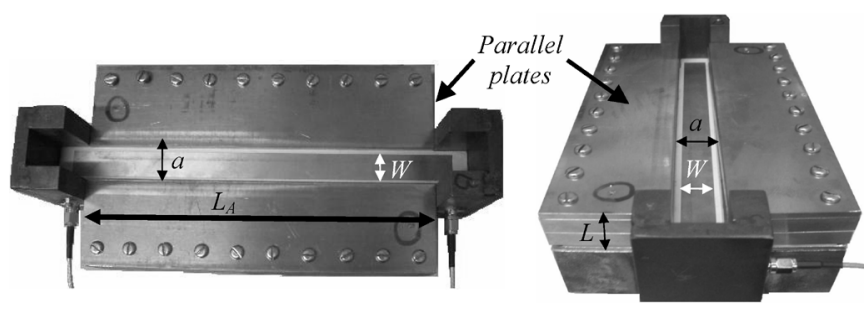

Fig. 16. Photograph of fabricated LSMLWA prototype.
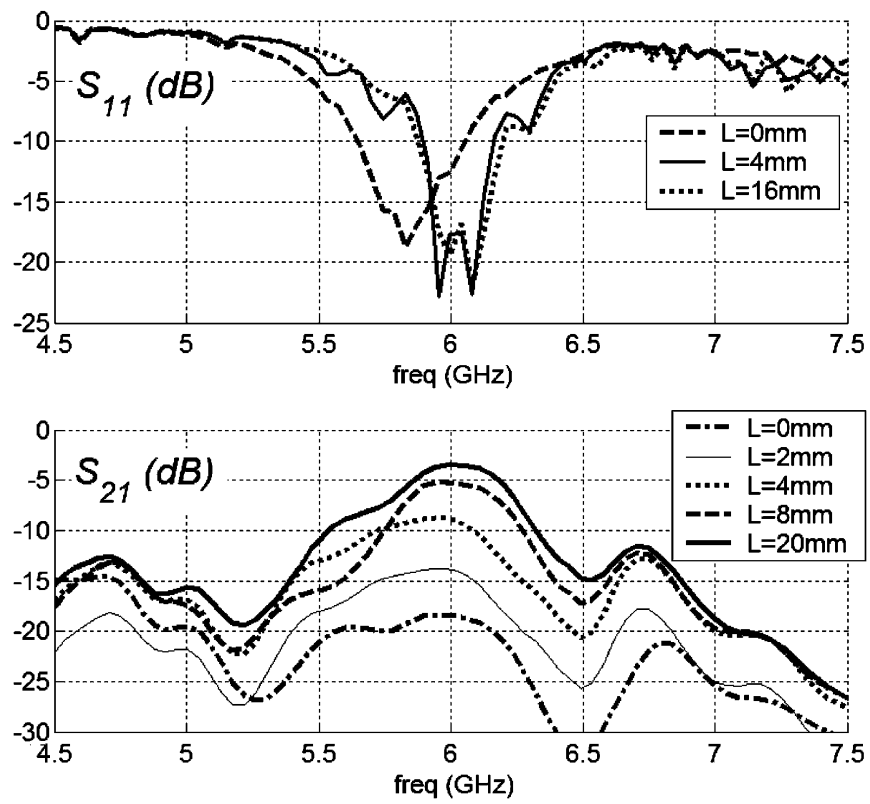

Fig. 17. Measured S-parameters for constructed prototype as a function of the parallel-plates height.

the results obtained for $S_{21}$, it is demonstrated that lower radiation occurs for higher values of $L$. Above $L=20 \mathrm{~mm}$, the transmission coefficient reaches the maximum value, which is lower than $0 \mathrm{~dB}\left(S_{21}=-3.5 \mathrm{~dB}\right)$ due to losses in dielectric and conductors.

The leakage rate can be estimated from the measured S-parameters, considering that losses can be split in two terms, one due to radiation leakage $\left(\alpha^{\mathrm{RAD}}\right)$ and the other due to ohmic losses $\left(\alpha^{\text {LOSSES }}\right)$

$$
\begin{aligned}
e^{-2\left(\alpha^{\mathrm{RAD}}+\alpha^{\mathrm{LOSSES}}\right) \cdot L_{A}}= & \left|S_{11}\right|^{2}+\left|S_{21}\right|^{2} \\
\frac{\alpha^{\mathrm{RAD}}}{k_{0}} & =-\frac{\ln \left(\left|S_{11}\right|^{2}+\left|S_{21}\right|^{2}\right)}{4 \pi \frac{L_{A}}{\lambda_{0}}} \\
& -\frac{\alpha^{\mathrm{LOSSES}}}{k_{0}} .
\end{aligned}
$$

Fig. 18 shows the estimation of the leakage-rate obtained from the measurements at $6 \mathrm{GHz}$ (using (9) with a computed value of $\alpha^{\text {LOSSES }} / k_{0}=0.017$ ), together with the theoretical simulations using MoM analysis technique. Good agreement is observed, confirming the novel technique to control the leakage rate in MLWAs.

Fig. 19 shows the measured normalized radiation pattern in the elevation plane at $6 \mathrm{GHz}$, for different parallel-plates heights. The observed pointing direction is in accordance with the simulations $\left(\theta_{m}=34^{\circ}\right)$. Moreover, $\theta_{m}$ is kept barely 


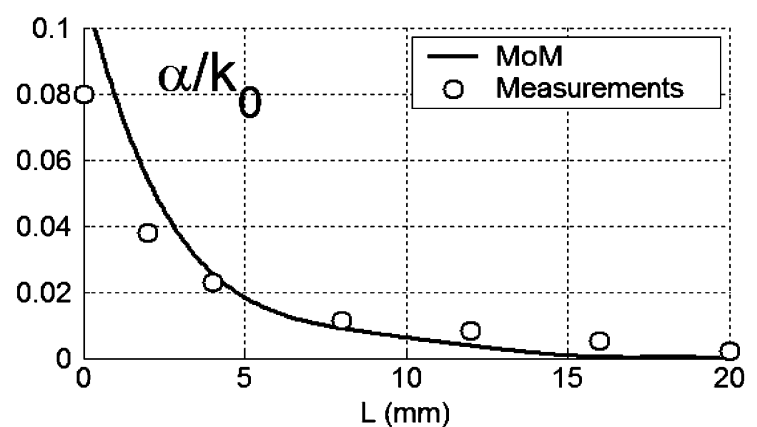

Fig. 18. Comparison between measured and simulated leakage rate at $6 \mathrm{GHz}$ as a function of the parallel-plates height.

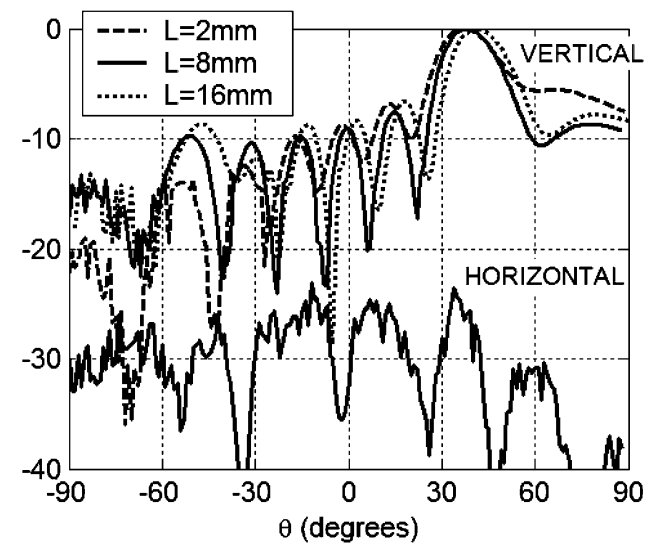

Fig. 19. Measured elevation radiation pattern at $6 \mathrm{GHz}$ for different parallelplates heights.

unchanged for different values of $L$, confirming the ability to control the leakage rate while barely affecting the designed pointing direction of the LWA. An increase can be observed in the reflected lobe (around $\theta_{m}=-50^{\circ}$ ) as $L$ is increased. This is caused by the leaky-wave reflected at the end of the antenna length, which amplitude is larger as $L$ is increased due to the lower leakage-rate. The polarization of the radiated fields is vertical ( $E_{\theta}$, according to Fig. 11), as it is common in MLWAs. The measured cross-pol component ( $E_{\theta}$, see Fig. 11) was found to be at least $20 \mathrm{~dB}$ below the vertical component for any value of $L$. In Fig. 19, the measured horizontal radiation pattern is shown for $L=2 \mathrm{~mm}$. For higher values of $L$, the cross-pol level is even lower, due to the aforementioned filtering-effect of the parallel-plates. The measured radiation efficiency of the structure (with $L_{A}=20 \mathrm{~cm}$ ) is $-4 \mathrm{~dB}$ for $L=2 \mathrm{~mm},-10 \mathrm{~dB}$ for $L=8 \mathrm{~mm}$ and $-20 \mathrm{~dB}$ for $L=16 \mathrm{~mm}$. These results are in accordance with the ability to control the amount of energy which is radiated or guided depending on the parallel-plate height $L$, as shown in Figs. 17 and 18 .

Fig. 20 shows the measured vertical radiation pattern in the azimuthal plane, confirming the existence of a single main beam for different values of $L$, compared to the dual-beam that appears for conventional second-higher order mode MLWA.

To finish this paper, it must be mentioned that a similar mechanism to control the leakage rate for nonradiative dielectric (NRD) guide technology [20], was proposed in [21], [22]. The proposed LSMLWA can be viewed as a printed-circuit version of the NRD antenna studied in [21], [22]. Due to the printed circuit technology proposed in this work, there is no

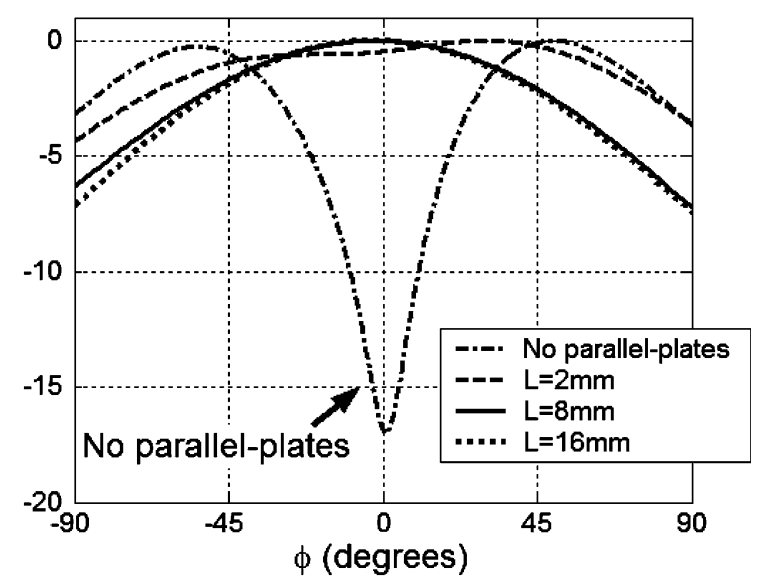

Fig. 20. Measured azimuthal radiation pattern at $6 \mathrm{GHz}$ for different parallelplates heights.

need to mechanize the NRD guide profile along the antenna length (as it was performed in [22]) therefore reducing the costs associated to mechanization of the waveguide. Moreover, the proposed LWA can be straightforwardly integrated with planar circuits. The sidewalls also help to prevent radiation from any unwanted surface-wave that could propagate in the dielectric substrate [4], [5].

\section{CONCLUSION}

In this paper, it has been demonstrated for the first time the ability to control the complex propagation constant of a microstrip leaky-wave mode, in order to conceive a novel MLWA with flexible control of both the pointing-direction and the leakage-rate. The proposed structure is based on the second higher order mode of the microstrip line and the addition of conductor sidewalls. Under the appropriate dimensions of the substrate and the separation of the parallel-plates, the fields of this mode become evanescent in the parallel-plate region. In this way, the leakage rate of the second higher order mode of the microstrip line can be controlled by modifying the parallel-plates height, with little influence on the pointing direction of the antenna. The proposed control mechanism has been illustrated by obtaining the dispersion curves of the leaky-mode complex propagation constant, using a MoMs approach. Moreover, it has been explained how this novel structure allows to obtain a single radiated beam in the azimuthal plane, in contrast to the dual-beam obtained in conventional microstrip second-higher order mode LWAs. Simulations of the three-dimensional structure and measurements of a fabricated prototype operating at $6 \mathrm{GHz}$ have been performed to confirm the advantages of the proposed LWA, obtaining very good agreement between theory and experiments. To the authors' knowledge, this is the first time that one can control the leakage rate of a microstrip leaky-mode, without strongly affecting its radiation direction. This is an essential characteristic to design practical LWAs with versatile radiation patterns.

\section{REFERENCES}

[1] W. Menzel, "A new-traveling wave antenna in microstrip," Arch. Elektron. Uebertrag. Tech., vol. 33, no. 4, pp. 137-140, Apr. 1979.

[2] H. Ermet, "Guiding and radiation characteristics of planar waveguides," Proc. Inst. Elect. Eng. Microw., Opt. Acoust., vol. 3, pp. 59-62, Mar. 1979 
[3] A. A. Oliner and K. Lee, "Microstrip leaky wave strip antennas," in Proc. Antennas and Propagation Society Int. Symp., Jun. 1986, vol. 24, pp. 443-446.

[4] A. A. Oliner, "Leakage from higher modes on microstrip line with application to antennas," Radio Sci., vol. 22, no. 6, pp. 907-912, Nov. 1987.

[5] J. S. Bagby, C. Lee, D. P. Nyquist, and Y. Yuan, "Identification of propagation regimes on integrated microstrip transmission lines," IEEE Trans. Microw. Theory Tech., vol. 41, no. 11, pp. 1887-1894, Nov. 1993.

[6] Z. Ma and E. Yamashita, "Space wave leakage from higher order modes on various planar transmission lines structures," in 1994 IEEE MTT-S Int. Microwave Symp. Dig, 1994, vol. 2, pp. 1033-1036.

[7] Y.-D. Lin and J.-W. Sheen, "Mode distinction and radiation-efficiency analysis of planar leaky-wave line source," IEEE Trans. Microw. Theory Tech., vol. 45, pp. 1672-1680, Oct. 1997.

[8] Y. D. Lin, J. W. Sheen, and C. K. C. Tzuang, "Analysis and design of feeding structures for microstrip leaky wave antenna," IEEE Trans. Microw. Theory Tech., vol. 44, no. 9, pp. 1540-1547, Sep. 1996.

[9] T. L. Chen and Y. D. Lin, "Aperture-coupled microstrip line leaky wave antenna with broadside mainbeam," Electron. Lett., vol. 34, no. 14, pp. 1366-1367, Jul. 1998.

[10] T. L. Chen and Y. D. Lin, "Microstrip leaky-wave antenna fed by short-end CPW-to-slot transition," Electron. Lett., vol. 35, no. 2, pp. 100-101, Jan. 1999.

[11] W. Hong, T.-L. Chen, C.-Y. Chang, J.-W. Sheen, and Y. D. Lin, "Broadband tapered microstrip leaky-wave antenna," IEEE Trans. Microw. Theory Tech., vol. 51, no. 8, pp. 1922-1928, Aug. 2003.

[12] G. J. Chou and C.-K. C. Tzuang, "Oscillator type active integrated antenna: The leaky mode approach," IEEE Trans. Microw. Theory Tech., vol. 44, pp. 2265-2272, Dec. 1996.

[13] C.-C. Lina and C. K. C. Tzuang, "A dual beam micro-CPW leaky-mode antenna," IEEE Trans. Antennas Propag., vol. 48, no. 2, pp. 310-316, Feb. 2000.

[14] Y.-D. Lin, P.-M. Chi, and T.-L. Chen, "Design of the feeding structures for the excitation of the microstrip line second higher order mode leakywave antenna," in IEEE Antenna and Propagation Society Int. Symp. Digest, 1997, pp. 1142-1145.

[15] T.-L. Chen, Y. D. Lin, and J.-W. Sheen, "Microstrip-fed microstrip second higher order leaky-mode antenna," IEEE Trans. Antennas Propag., vol. 49, no. 6, pp. 855-857, Jun. 2001.

[16] T.-L. Chen and Y. D. Lin, "Aperture coupled microstrip second higher order leaky-mode antenna," in Proc. APMC'2001, 2001, vol. 49, no. 6, pp. 1060-1063.

[17] A. A. Oliner, "Leaky-wave antennas," in Antenna Engineering Handbook, R. C. Johnson, Ed., 3rd ed. New York: McGraw-Hill, 1993, ch. 10.

[18] J. L. Gómez and A. A. Melcón, "Radiation analysis in the space domain of laterally-shielded planar transmission lines. Part I: Theory," Radio Sci., vol. 39, pp. 1-11, Jun. 2004, RS3005.

[19] J. L. Gómez, F. D. Quesada, and A. A. Melcón, "A full-wave spacedomain method for the analysis of leaky-wave modes in multilayered planar open parallel-plate waveguides," Int. J. RF Microw. Comput. Aided Eng., vol. 15, no. 1, pp. 128-139, Jan. 2005.

[20] T. Yoneyama and S. Nishida, "Nonradiative dielectric waveguide for millimeter-wave integrated circuits," IEEE Trans. Microw. Theory Tech., vol. 29, pp. 1188-1192, Nov. 1981.

[21] A. Sanchez and A. A. Oliner, "A new leaky waveguide for millimeter waves using nonradiative dielectric (NRD) waveguide-Part I: Accurate theory," IEEE Trans. Microw. Theory Tech., vol. 35, no. 8, pp. 737-747, Aug. 1987.

[22] A. Sanchez and A. A. Oliner, "A new leaky waveguide for millimeter waves using nonradiative dielectric (NRD) waveguide-Part II: Comparison with experiments," IEEE Trans. Microw. Theory Tech., vol. 35, no. 8, pp. 748-752, Aug. 1987.

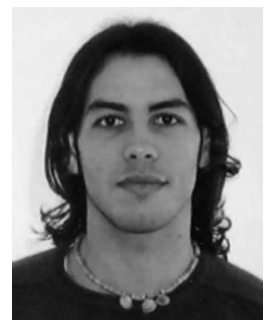

José Luis Gómez-Tornero (M'06) was born in Murcia, Spain, in 1977. He received the Telecommunications Engineer degree from the Polytechnic University of Valencia (UPV), Valencia, Spain, in 2001, and the Ph.D. degree in Telecommunication Engineering (laurea cum laude) from the Technical University of Cartagena (UPCT), Cartagena, Spain, in 2005.

In 1999, he joined the Radiocommunications Department, UPV, as a Research Student, where he was involved in the development of analytical and numer- ical tools for the study and automated design of microwave filters in waveguide technology for space applications. In 2000, he joined the Radio Frequency Division, Industry Alcatel Espacio, Madrid, Spain, where he was involved with the development of microwave active circuits for telemetry, tracking and control (TTC) transponders implicated in many different spatial missions for the European Space Agency (ESA), National Aeronautics Space Administration (NASA) and other Space Agencies. In 2001, he joined the Technical University of Cartagena, Spain, as an Assistant Professor, where he is currently developing his teaching activities. Since October 2005, he holds the position of Vice Dean for Students and Lectures Affairs in the Telecommunication Engineering Faculty at the UPCT. His scientific research is focused on the analysis and design of leaky-wave antennas for millimeter waveband applications and the development of numerical methods for the analysis of novel passive radiating structures in planar and waveguide technologies. His scientific interests also include the study of active devices for microwave and millimeter wavebands, such as oscillators and active antennas.

José Luis Gómez Tornero received the National Award from the EPSONIbérica Foundation for the Best Ph.D. Project in the field of technology of information and communications (TIC), in July 2004. In June 2006, he received the Vodafone Foundation-COIT/AEIT (Colegio Oficial de Ingenieros de Telecomunicació) Award for the Best Spanish Ph.D. Thesis in the area of advanced mobile communications technologies, this thesis was also awarded Best Thesis in the area of Electrical Engineering, by the Technical University of Cartagena in December 2006

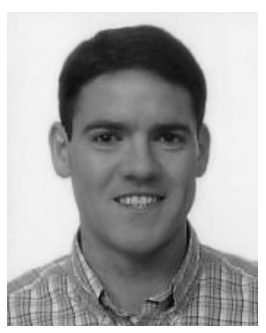

David Cañete-Rebenaque ( $\left.\mathrm{S}^{\prime} 04\right)$ was born in Valencia, Spain, in 1976. He received the Telecommunications Engineer degree from the Technical University of Valencia, Valencia, Spain, in 2001, and he is currently working toward the Ph.D. degree at the Technical University of Cartagena (UPCT), Cartagena, Spain.

During 20001, he worked as an RF engineer for a mobile communication company. In 2002, he joined the Communications and Information Technologies Department, (UPCT). His research interests include the analysis and design of microwave circuits and active antennas.

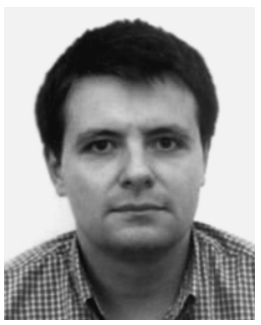

Alejandro Alvarez-Melcón (M'99) was born in Madrid, Spain, in 1965. He received the Telecommunications Engineer degree from the Polytechnic University of Madrid (UPM), Madrid, Spain, in 1991, and the Ph.D. degree in electrical engineering from the Swiss Federal Institute of Technology, Lausanne, Switzerland, in 1998.

In 1988 he joined the Signal, Systems and Radiocommunications Department, UPM, as a Research Student, where he was involved in the design, testing, and measurement of broadband spiral antennas for electromagnetic measurements support (EMS) equipment. From 1991 to 1993, he joined the Radio Frequency Systems Division, European Space Agency (ESA/ESTEC), Noordwijk, The Netherlands, where he was involved in the development of analytical and numerical tools for the study of waveguide discontinuities, planar transmission lines, and microwave filters. From 1993 to 1995, he joined the Space Division, Industry Alcatel Espacio, Madrid, Spain, and he worked at the ESA, where he collaborated in several ESA/ESTEC contracts. From 1995 to 1999, he joined the Swiss Federal Institute of Technology, École Polytechnique Fédérale de Lausanne, Lausanne, Switzerland, where he worked in the field of microstrip antennas and printed circuits for space applications. In 2000, he joined the Technical University of Cartagena, Spain, where he is currently developing his teaching and research activities.

Dr. Alejandro Alvarez Melcón received the Journée Internationales de Nice sur les Antennes (JINA) Best Paper Award for the Best Contribution to the JINA'98 International Symposium on Antennas, and the COIT/AEIT (Colegio Oficial de Ingenieros de Telecomunicación) award for Best Ph.D. Thesis in Basic Information and Communication Technologies. 